\title{
The Observance of the International Acts from the Albanian Penal and Procedurial Penal Legislation in Consideration to the Juvenile Protection
}

\author{
Bledar Mustafaraj \\ Prosecutor, Public Prosecutor's Office of the Judicial Region Elbasan \\ PhD branch, European University of Tirana
}

\section{Doi:10.5901/mjss.2013.v4n9p175}

\section{Abstract}

The juveniles' right bore and is getting developed as a very important branch of the law. The principals that should be followed to respect the juveniles' rights in the normative and institutional aspect dwell there. The foundations of these principals are placed in many international acts, specific for the juveniles. This work aims to fulfill a comparative study between the international legislation and that of the Albanian state, in protection of the juvenile. The aim is a two-folded one: firstly to observe up to what stage the Albanian legislation guaranties this protection keeping in mind the international standards and secondly to identify in the specific internal laws of the Albanian government the provisions that ensure the juvenile protection. It cannot be spoken of a mere juvenile justice system in Albania that not only can recognize their rights but also execute them accurately. The first step made toward this direction is the recognition of these rights in the Albanian legislation, the ratification of different international acts in protection of the juvenile. The circle of the rights presented to the children in the internal juridical order in Albania is growing. It is of a great importance that even in Albanian the branch of the juveniles' rights can be observed as a special branch of the justice in general with its own principals accompanied by a convenient judicial practice. This, in order that the juvenile (mainly the delinquent juvenile), can have reliefs to grow up and get developed within the social community where he lives. The internal laws that will be analyzed are: The Constitution, The Penal Code and The Code of Penal Procedure of the Albanian Republic.

Keywords: juvenile, juveniles' law, comparison of legislations, guaranties and the assured protection within the internal laws.

\section{Introduzione}

La delinquenza minorile rappresenta un fenomeno sociale molto importante e un problema criminologico affrontato in continuazione da studiosi ed esperti1. La società albanese, nel suo attuale stato di sviluppo, si trova di fronte a una dualità di generazioni, ove i giovani d'oggi stanno crescendo con una mentalità diversa da quella delle generazioni precedenti. I fattori socio - economici sono evoluti e cambiati drasticamente, soprattutto dopo gli anni ' 90 , contribuendo alla trasformazione del fenomeno di delinquenza minorile rispetto a come si presentava prima. In queste condizioni, è di massima importanza adottare un sistema legale appropriato per il trattamento del minore delinquente e del fenomeno della delinquenza minorile in generale.

Nel corso di questi due decenni, lo stato albanese ha elaborato la sua legislazione, introducendo principi e strumenti che, benché operanti all'interno del processo penale ordinario, consentono un approccio del minore indagato e imputato orientato alla finalità di recupero mediante la sua rieducazione ed il suo reinserimento sociale. Di conseguenza, l'insieme dei diritti che sono riconosciuti ai minori nell'ordinamento giuridico interno è aumentato in modo considerevole, specialmente in seguito alla ratifica degli atti internazionali a tutela dei diritti dei minori dallo Stato albanese.

Comunque, in Albania, ancora non si può parlare di un vero e proprio sistema di giustizia minorile che non solo riconosca i diritti dei minori, ma che ne garantisca anche l'applicazione.

\section{Una breve panoramica storica della legislazione penale riguardante i minori in Albania}

Il concetto "minore" nella legislazione penale albanese, ha subito dei cambiamenti frequenti a secondo le circostanze storiche.

1. La legge penale ottomana, sanciva nell'articolo 40:

- II reo che non ha compiuto i tredici anni non puo essere condannato penalmente, ma si pone sotto cura

${ }^{1}$ Art. 8 della Legge n. 253 del 24 dicembre 1946 "Disposizioni generali penali", entrato in vigore dopo la seconda guerra mondiale. 
dei genitori, tutori, oppure si rinchiude in un centro di correzione;

- II reo dell'età dai tredici fino ai quindici anni, può essere condannato da 5 - 10 anni di carcere quando comette un delitto che è punibile con la pena di morte, l'ammanettamento o l'ergastolo;

- Per il reo dell'età dai quindici fino agli anni diciotto, la pena più grave non può superare i 15 anni di reclusione e quella più bassa non può superare la metà della pena prevista, a secondo della disposizione.

2. II codice penale del Regno d'Albania del 1928 sanciva negli artt. 54 e 56 l'età minima per l'imputabilità quella di dieci anni e l'età massima quella di diciotto anni. L'art. 54, comma 1, di questo codice stabiliva che "non è imputabile colui che nel momento della commissione del reato non ha compiuto dieci anni". Si applicavano misure sanzionatorie diverse per i minori colpevoli di reato dell'età dai dieci fino ai quindici anni e diverse per i minori colpevoli di reato dell'età dai quindici fino ai diciotto anni.

3. La legge n. 253 del 24 dicembre 1946 in materia di "Disposizioni generali penali", stabiliva l'età minima di quattordici anni per considerare un minore imputabile. Per i reati di rapina, lesioni gravi, omicidio, i reati contro lo stato e il popolo, il sabotaggio economico, era imputabile anche il minore di dodici anni².

4. II codice penale del 1948 considerava imputabile il minore che aveva raggiunto l'età di quattordici anni. Questo codice ha stabilito oltre la minore età anche la capacità di intendere e di volere come elemento necessario dell'imputabilità. In mancanza di questa capacità, il soggetto non era considerato imputabile 3 .

5. Il codice penale del 1952 aveva previsto l'età minima di quattordici anni per considerare un minore imputabile. Se il minore degli anni quatordici era considerato socialmente pericoloso, poteva essere sottoposto alle misure di rieducazione $e^{4}$. L'art. 19 di questo codice sanciva il divieto della pena di morte per i minori che nel momento in cui avevano commesso il reato, non avevano compiuto i diciotto anni.

6. II codice penale del 1977 , aveva stabilito l'età minima di 14 anni per essere considerato imputabile un minore ${ }^{5}$. L'art. 12 di questo codice sanciva il divieto della pena di morte per i minori che nel momento in cui avevano commesso il reato, non avevano compiuto i diciotto anni.

7. II codice penale vigente, entrato in vigore il 1 giugno 1995 (art. 12) sancisce:

Non è imputabile chi, nel momento in cui ha commesso il delitto, non aveva compiuto i quattordici anni.

Non è imputabile chi, nel momento in cui, ha commesso la contravvenzione penale, non aveva compiuto i sedici annit.

Si può notare che la legislazione penale albanese nel corso degli anni ha sempre stabilito un'età minima riguardo all'imputabilità del minore. II minore colpevole di reato non è stato soggetto della pena di morte. Si nota anche che l'età minima per essere considerati imputabili i minori autori di reato, è stata generalmente quella di quattordici anni. Questa minima età è stata stabilita tenendo in conto fattori tali come la capacità intellettuale dei minori, la loro maturità e le condizioni sociali. La valutazione di questi fattori ha servito per stabilire l'abilità di un minore di riconoscere quale azione comporta un reato e quale no.

\section{I diritti dei minori e rilevanza costituzionale}

Dopo la caduta del sistema comunista, l'adozione del codice penale e quello di procedura penale nel 1995 e della nuova Costituzione nel 1998, in Albania finalmente si è iniziato a parlare anche di diritti dei minori, diritti menzionati prima di tutto nella Costituzione albanese, entrata in vigore il 28 novembre 1998, la quale conforme all'art. 116 della stessa, costituisce la legge fondamentale dello Stato, sul rispetto della quale devono ispirarsi tutte le leggi dello Stato ${ }^{7}$.

La rilevanza costituzionale del minore trova il suo fondamento nell'art. 3 della Costituzione della Repubblica d'Albania, secondo cui i diritti umani (comprese quelle dei minori) sono valori fondanti dello Stato che prende in carico di proteggere e rispettare.

La Costituzione albanese riconosce e garantisce tali diritti e libertà dell'uomo, sia come singolo sia all'interno delle

\footnotetext{
${ }^{2}$ Artt. 7 e 8 di questo codice penale.

${ }^{3}$ Art. 6 del codice penale del 1952.

${ }^{4}$ Questa minima età era prevista dall'art. 7 di questo codice penale.

5 Tale disposizione normativa è del tutto coerente con l'art. 1 della Convenzione sui Diritti dell'Infanzia.

${ }^{6}$ In conformità a quest'articolo, al primo livello della gerarchia delle fonti del diritto nell'ordinamento giuridico albanese si pone la Costituzione e al secondo, i trattati internazionali. In conformità all'art. 122 della Costituzione albanese, i trattati internazionali ratificati hanno efficacia immediata.

${ }^{7}$ Articolo 54 della Costituzione della Repubblica d'Albania.
} 
formazioni sociali ove sviluppa la sua personalità. Una di queste formazioni è la famiglia, la quale prende cura del minore dalla nascita fino alla sua maturità, per far in modo che lui sia capace di vivere la vita in maniera indipendente ${ }^{8}$. Ai minori si garantisce una protezione speciale da parte dello Stato. Ė questo momento di massima importanza perché, quanto più lo Stato prende cura dei minori e della loro educazione, maggiori saranno i profitti della società, perché i minori di oggi saranno i cittadini di domani. Per raggiungere questo scopo è necessario costruire un sistema legislativo efficace e non solo, ma anche le strutture necessarie all'interno delle quali le norme sono materializzate. Questi principi di base sono specificati in altre leggi come il codice di famiglia della Repubblica d'Albania, il quale nella sua interezza è guidato dal principio del superiore interesse del minore, principio base della Convenzione sui Diritti dell'Infanzia in conformità all'articolo 3 della stessa9 .

In conformità con la normativa internazionale, specialmente con le convenzioni ILO $138^{10}$ e $182^{11}$, l'articolo 54 , comma 3, della Costituzione albanese elenca una serie di divieti e garanzie in materia di protezione dei bambini dalla violenza, abusi, sfruttamento e l'utilizzo per il lavoro, soprattutto sotto l'età minima, con lo scopo di procurare ai minori un normale processo di crescita, sia fisico sia psicologico e morale. A questo proposito, il codice di lavoro dell'Albania specifica in maniera dettagliata le garanzie per la tutela dei minori nei rapporti di lavoro.

Un altro punto importante è il riconoscimento del diritto all'istruzione che è legato direttamente all'educazione dei minori (art. 57 della Costituzione). II sistema d'istruzione attuale albanese rispetta i diritti dei minori sanciti nelle leggi nazionali e nella normativa internazionale, in conformità anche agli artt. 20 e 21 delle Linee Guida delle Nazioni Unite sulla Prevenzione della Delinquenza Minorile del 14.12.199012.

La Costituzione albanese presta particolare attenzione ai casi di privazione della libertà e la necessità di rispettare i diritti delle persone private della libertà. Così l'art. 27, comma 2, della Costituzione in materia di casi della limitazione della libertà di una persona, afferma che la libertà di una persona non può essere limitata, ad eccezione dei seguenti casi:

d) la vigilanza di un minore a fini d'istruzione o per scortarlo a disposizione dell'autorità competente.

Quest'articolo è in conformità all'art. 5 della Convenzione Europea per la Salvaguardia dei Diritti dell'Uomo e delle Libertà Fondamentalii3 ${ }^{3}$. Inoltre, i casi di restrizione della libertà dei minori sono previsti in leggi speciali come il codice penale e il codice di procedura penale. II codice penale prevede anche la protezione giuridico-penale del minore dai reati diretti contro di lui.

\section{Analisi comparativa della normativa internazionale a tutela dei minori e la legislazione penale albanese}

L'imputabilità, la differenziazione nel trattamento durante il procedimento penale e nel sistema delle pene per i minorenni e per gli adulti, la specializzazione dei soggetti istituzionali che operano nel processo minorile, rappresentano i principi della normativa internazionale a tutela dei minori sui quali prende forma la giustizia minorile.

L'art. 40, comma 4, della Convenzione sui Diritti dell'Infanzia (cosi come gli artt. 52-59 delle Linee guida di Riyadh e l'art. 2, comma 3, delle Regole Minime delle Nazione Unite sull'Amministrazione della Giustizia Minorile ${ }^{14}$ ) stabilisce che Gli Stati Parti si sforzano di promuovere l'adozione di leggi, di procedure, la costituzione di autorità e di istituzioni destinate specificamente ai minori sospettati, accusati o riconosciuti colpevoli di aver commesso reato.

Il legislatore albanese, dopo aver ratificato questa Convenzione ${ }^{15}$, ha incluso nel codice penale e quello di procedura penale numerosi principi, i quali forniscono sufficienti garanzie per i minori e creano un trattamento differenziato a loro favore quando sono soggetti di un processo penale. È un fatto di massima importanza poiché queste norme obbligano le autorità giudiziarie di procedimento penale di trattare i minori delinquenti in maniera diversa dai maggiori. Comunque, non esiste ancora in Albania un sistema di giustizia minorile in conformità con la normativa internazionale (Convenzione sui Diritti dell'Infanzia, le Regole Minime delle Nazione Unite sull'Amministrazione della

\footnotetext{
${ }^{8}$ La promozione e la tutela dei diritti dei minori è un obiettivo dell'Unione in conformità all'art. 24 della Carta dei diritti fondamentali dell'Unione Europea.

${ }^{9}$ Resa esecutiva in Albania con legge n. 8086 del 13.03.1996, pubblicata nella gazzetta ufficiale n. 6, p. 261.

${ }^{10}$ Resa esecutiva in Albania con legge n. 8774 del 23.04.2001, pubblicata nella gazzetta ufficiale n. 23, p. 708.

${ }_{11} \mathrm{Da}$ ora in poi citato come Linee Guida di Riyadh.

${ }^{12}$ Resa esecutiva in Albania con legge n. 8137 del 31.07.1996, pubblicata nella gazzetta ufficiale n. 20.

${ }^{13} \mathrm{Da}$ ora in poi citato come Regole di Pechino.

${ }^{14} \mathrm{La}$ Convenzione sui Diritti dell'Infanzia è stata resa esecutiva dal Parlamento Albanese il 27.02.1992. Non si conosce il numero della gazzetta ufficiale, dove la legge della ratifica è stata pubblicata.

${ }^{15}$ Art. 290 del codice di procedura penale albanese.
} 
Giustizia Minorile, le Linee Guida delle Nazioni Unite sulla Prevenzione della Delinquenza Minorile e le Regole delle Nazioni Unite per la Protezione dei Minori Privati della Libertà), a cui fare ricorso in alternativa al giudizio.

In questa parte del lavoro ho cercato di fare un'analisi comparativa tra i principi della normativa internazionale a tutela dei minori e la legislazione penale albanese (codice penale e codice di procedura penale) riguardo ai diritti dei minori autori di reato.

L'art. 40, comma 4, lettera (a) della Convenzione sui diritti del minore prevede la definizione di una età sotto la quale i minorenni devono essere considerati non capaci di infrangere la legge penale. Lo stesso principio è stabilito dall'art. 4 delle Regole di Pechino, ove è richiesto di stabilire un minimo di età per l'imputabilità tenendo conto della maturità affettiva, mentale e intellettuale. La definizione di questa età è prevista dall'art. 12 del codice penale dell'Albania, sopra-citato. L'art. 41 del codice di procedura penale albanese obbliga il pubblico ministero e il giudice di accertare l'età della persona, poiché, per l'imputabilità occorre provare che la persona sottoposta alle indagini abbia raggiunto i quattordici anni se ha commesso un delitto e i sedici anni nel caso in cui ha commesso una contravvenzione penale. Quando vi è incertezza sulla minore età, l'autorità processuale dispone, anche d'ufficio, la perizia. La minore età è presunta, qualora, anche dopo la perizia, permangano dubbi. Per l'autorità processuale, l'accertamento dell'età è importante in primo luogo per decidere se il soggetto che ha commesso reato sia minore o meno e in secondo luogo se trattasi di minore imputabile o meno. Nel caso in cui si stabilisce che il minore non ha raggiunto l'età minima per essere considerato imputabile, l'organo d'accusa, ossia, il pubblico ministero, dispone tramite decreto motivato a non esercitare l'azione penale ${ }^{16}$. II codice di procedura penale dell'Albania non prevede l'accertamento della capacità di intendere e di volere oltre all'accertamento dell'età. Questa capacità non è stata prevista neanche dalle disposizioni generali del codice penale.

L'art. 40, comma 2, della Convenzione sui Diritti dell'Infanzia stabilisce delle garanzie che devono essere riconosciute ai minori dagli Stati Parti. In conformità a quanto stabilisce questa convenzione, l'art. 7 delle Regole di Pechino sancisce che ai giovani devono essere assicurate sempre garanzie procedurali di base quali la presunzione di innocenza, il diritto alla presenza del genitore e del tutore, il diritto alla notifica delle accuse, il diritto al confronto e all'esame incrociato dei testi, il diritto a non rispondere e il diritto di appello. Paragonando in maniera dettagliata le garanzie stabilite dagli atti internazionali a quelle stabilite dalla legislazione penale albanese:

- II minore non può essere perseguito, accusato o riconosciuto colpevole di reato penale a causa di azioni o di omissioni che non erano vietate dalla legislazione nazionale 0 internazionale nel momento in cui furono commessi. Questa garanzia trova espressione nell'art. 2 del codice penale albanese dove è sancito quanto segue: "Nessuno può essere riconosciuto colpevole di un reato che non è già previsto nella legge come delitto o contravvenzione penale. Nessuno può essere punito con pene che non sono stabilite dalla legge".

- II minore deve essere considerato innocente fino a che la sua colpevolezza non sia stata legalmente provata. Questo principio è previsto dall'art. 4 del codice di procedura penale albanese, secondo il quale, l'imputato si ritiene innocente sino alla condanna definitiva.

- II minore deve essere informato sollecitamente e direttamente delle accuse a suo carico, o all'occorrenza, tramite i suoi genitori o tutori. Questo principio è rispettato dall'art. 34, comma 1, del codice di procedura penale albanese che sancisce il diritto dell'imputato di essere informato sulle accuse a suo carico, però tale articolo si riferisce all'imputato in generale e non all'imputato minorenne in particolare.

- II minore deve avere la propria causa istruita senza indugi da un organo giudiziario o da un'autorità competente, indipendente e imparziale in un'udienza equa e conforme alla legge. II codice di procedura penale albanese stabilisce che il tribunale è indipendente, imparziale, precostituito per legge, le cui decisioni si prendono di seguito alle prove analizzate in udienza (art. 3 di questo codice).

- II minore non deve essere obbligato a testimoniare 0 a confessarsi colpevole. Gli artt. 38, comma 2, e 166 del codice di procedura penale d'Albania, riferendosi all'imputato in generale, riconoscono il diritto di costui di non sottoporsi a metodi che influiscono sulla libertà di agire e di pensare e di essere interrogato con il suo consenso.

- II minore ha il diritto di interrogare o far interrogare i testimoni a carico e ottenere la comparizione e la deposizione dei testimoni a discarico, in condizioni di uguaglianza. Nella fase del giudizio, secondo il codice di procedura albanese, le parti chiedono le prove le quali si formano come tali in sede dibattimentale (artt. 151, comma 2, e 359 di questo codice).

- II minore ha il diritto di avere assistenza legale. Questo principio, di estrema importanza, è previsto dall'art. 35,

${ }^{16}$ Corte Costituzionale, 28.02.2000, n. 7, in Vendime të Gjykatës Kushtetuese të RSH, Mihal Duri e Re, Tiranë 2002, pp. 14-19. 
comma 1, del codice di procedura penale della Repubblica d'Albania, riferendosi però all'imputato in generale. Inoltre, il codice di procedura penale dell'Albania prevede un'ulteriore garanzia riconoscendo che l'assistenza legale per i minori di 18 anni è obbligatorio (art. 49, comma 2, di questo codice). In quanto a questo diritto, l'imputato A. L, in età minorenne ha ricorso alla Corte Costituzionale della Repubblica d'Albania chiedendo di dichiarare non conforme ai principi della Costituzione la delibera del giudice di primo grado del Tribunale Distrettuale di Argirocastro, della Corte d'Apello d'Argirocastro e del Colegio Penale della Corte Suprema di Albania, perché era stato interrogato dalla Polizia Giudiziaria senza un avvocato. La Corte Costituzionale ha costatato che al minore non era assiccurato l'assistenza legale al primo momento dell'interrogatoria, ma durante la stessa. In questo modo la Corte ha stabilito che al minore non era stato negato il diritto di essere assistito da un diffensore. Le dichiarazioni dell'imputato minorenne durante l'interrogatorio senza assistenza legale sono uguali a quelle date nella presenza del diffensore, per questo motivo la violazione accaduta all'inizio del processo penale non è di quel grado da violare il giusto processo ${ }^{17}$. Non sarei d'accordo con questa decisione della corte costituzionale perché la violazione accaduta all'inizio del processo è di tale natura che infrange i diritti riconosciuti al minore processato penalmente dalla normativa internazionale a tutela dei minori. Soprattutto, violazioni del genere non possono essere giustificate in un paese come l'Albania che tuttavia non ha costruito un sistema vero e proprio di giustizia minorile.

- II minore ha il diritto di essere assistito dai genitori o dai tutori. L'art. 35 del codice di procedura penale albanese stabilisce il diritto dell'imputato minorenne di essere assistito dai genitori o da altre persone da lui indicate durante il processo penale a suo carico.

- II minore ha il diritto di avvalersi dell'assistenza gratuita di un interprete, qualora non sia in grado di parlare 0 di comprendere la lingua utilizzata. Tale principio è stato prevvisto dagli artt. 8, comma 2, e 123 del codice di procedura penale albanese. Riferendosi all'imputato in generale, questi articoli riconoscono il diritto dell'imputato di essere assistito da un interprete per essere informato sulle accuse a suo carico.

- $\quad$ II minore ha il diritto di avere il pieno rispetto della sua vita privata in tutte le fasi del procedimento. In quanto a questo principio la legge albanese prevede delle garanzie sull'identità e le fotografie dell'imputato 0 del testimone minorenne (art. 103 del codice di procedura penale), sia durante la fase delle indagini preliminari sia quella del giudizio. Durante quest'ultima, il giudice può disporre di vietare al pubblico e alla stampa l'accesso alla sala d'udienza quando è ritenuto opportuno durante la testimonianza dei minori (art. 340 del codice di procedura penale). Questo principio è stabilito anche dall'art. 6, comma 1, della Convenzione Europea per la Salvaguardia dei Diritti dell'Uomo e delle Libertà Fondamentali e in quanto a questo, la legislazione albanese è in conformità con gli atti internazionali riguardanti questi diritti. Il codice di procedura penale d'Albania, art. 361, presta attenzione al momento in cui vengono interrogati i testimoni minorenni, nella fase del giudizio. Tenendo conto della loro età e dal modo in cui percepiscono i fatti, quest'articolo stabilisce che il testimone minorenne deve essere interrogato dal giudice che presiede la sessione del tribunale. II giudice può essere assistito durante l'interrogazione da un familiare del minorenne oppure da uno specialista in materia di educazione dei bambini (un insegnante, un assistente sociale 0 uno psicologo specializzato nel trattamento dei minori). La legge penale presta attenzione allo stato psicologico del minore perché non si nuoci dalle domande delle parti in giudizio. Quando la domanda diretta non nuoce il minore psicologicamente, questa potrebbe essere rivolta secondo la procedura normale.

- Se considerato colpevole di aver infranto la legge penale, il minore ha il diritto di presentare appello contro tale pronunciamento e qualsiasi provvedimento ad esso conseguente presso un'istanza giuridica 0 a un'attività competente, indipendente e imparziale di grado più elevato, come stabilito dalla legge. Queste garanzie sono previste dagli artt. 410, commi 1 e 3, del codice di procedura penale dell'Albania. La Corte Suprema della Repubblica d'Albania riferendosi al caso dell'imputato minorenne B. H. assistito da un difensore nominato d'ufficio dal tribunale di primo grado di Durrazzo ha concluso: "Non è più necessario che quest'ultimo tramite atto di nomina del difensore dell'imputato, presenti appello contro la sentenza di primo grado. Per ricorrere all'appello è sufficiente che il difensore sia stato nominato d'ufficio durante il processo penale come tale ${ }^{18 "}$.

L'art. 10 delle Regole di Pechino, stabilisce che quando un giovane viene arrestato, i suoi genitori o il tutore dovranno essere immediatamente informati del suo arresto e, quando ciò non è possibile, sarà data loro notizia nel più breve tempo. L'art. 255, comma 4, del codice di procedura penale d'Albania stabilisce la stessa garanzia, obbligando la

${ }^{17}$ Sentenza delle Sezioni Unite della Corte Suprema d'Albania, 28.1.1999, n. 30, in www.gjykataelarte.gov.al, voce Vendime Unifikuese.

18 Tale regola è stabilita anche dalle Regole Penitenziarie Europee oppure Raccomandazione n. R (2006) 2. 
polizia giudiziaria di informare immediatamente i genitori o il tutore dell'arresto 0 il fermo del minore. $\| 3^{\circ}$ comma dello stesso articolo riconosce la competenza del pubblico ministero di ordinare la detenzione del minore arrestato presso il suo domicilio 0 in un altro luogo protetto.

L'art. 13.1 delle Regole di Pechino stabilisce che la detenzione preventiva può essere una misura usata come ultimo mezzo e la sua durata deve essere la più breve possibile, offrendo altre possibilità quali la sorveglianza, un aiuto molto attento 0 l'affidamento ad una famiglia, a un istituto 0 ad un focolare educativo ${ }^{19}$. Nel codice di procedura penale dell'Albania queste garanzie non sono riconosciute esplicitamente ed esclusivamente al minore detenuto, non si prevedono i limiti per la durata della custodia cautelare in carcere che siano più favorevoli in confronto ai detenuti adulti, soprattutto mancano le misure alternative alla detenzione in materia di misure cautelari. La legge n. 9090/2003 in materia di "La mediazione finalizzata alla conciliazione delle controversie" ha creato la possibilità di gestire i conflitti derivanti da compimento di contravvenzioni penali senza ricorrere al Tribunale (la mediazione penale, soprattutto per i minori, non è ancora applicata in Albania).

II $4^{\circ}$ comma dell'art. 13 delle Regole di Pechino stabilisce che i minori detenuti dovrebbero stare separatamente dagli adulti, mentre il $5^{\circ}$ comma dello stesso articolo riconosce ai minori detenuti il diritto alla cura sociale, educativa, professionale, psicologica, sanitare e fisica in maniera personalizzata. L'art. 229 del codice di procedura penale dell'Albania indica al tribunale di non interrompere il processo educativo in atto del minore nel momento in cui applica la misura cautelare.

L'art. 16 delle Regole di Pechino stabilisce che l'autorità competente deve indagare sulle condizioni di vita, i precedenti del minore e le circostanze in cui è stato commesso il reato. In conformità a questa regola, l'autorità competente in Albania, il pubblico ministero o il giudice, acquisisce elementi circa le condizioni e le risorse personali, familiari e sociali dell'imputato minorenne al fine di accertarne l'imputabilità e il grado di responsabilità, valutare la rilevanza sociale del fatto e disporre le adeguate misure penali (art. 42 del codice di procedura penale dell'Albania).

II $22^{\circ}$ articolo delle Regole di Pechino punta nella specializzazione dei soggetti che operano nel sistema minorile. Combinati, gli artt. 13 e 81 del codice di procedura penale dell'Albania stabiliscono che l'azione penale nei confronti dei minorenni verrà effettuato nelle sezioni per i minorenni presso i tribunali di primo grado, stabiliti con decreto del Presidente della Repubblica ${ }^{20}$. In conformità a questo principio, non solo i giudici, ma anche i pubblici ministeri e gli ufficiali della polizia giudiziaria che conducono le indagini preliminari su un minorenne, devono avere una specifica qualificazione non solo nel piano giuridico, ma anche in quello sociologico, psicologico, criminologico e in altre scienze che studiano il comportamento umano. Solo in questo modo si potrebbero rispettare pienamente i diritti dei minori. La legislazione penale albanese attuale non sancisce la necessità delle competenze professionali e formazione del personale che si occupa di minori, ancor di meno si può parlare di queste competenze per altre figure come gli avvocati difensori.

In conformità all'art. 20 delle Regole per la Protezione dei Minori Privati della Libertà ${ }^{21}$ sono gli artt. 11, comma 2, e 464 del codice di procedura penale dell'Albania. Al minore che ha commesso un reato, è garantito di essere dichiarato colpevole e condannato solo tramite la sentenza del tribunale competente (questa garanzia è riconosciuta all'imputato in generale e non si menziona il minore in maniera particolare). La sentenza si mette in esecuzione in seguito all'ordine emanato dal pubblico ministero. Di ordine, i minori scontano le pene di reclusione nei carceri minorili22.

L'art. 37 della Convenzione sui Diritti dell'Infanzia obbliga gli Stati Parti di garantire che, né la pena capitale, né l'ergastolo senza possibilittà di liberazione debbano venire irrogate per reati commessi da persone in età inferiore ai 18 anni. La stessa garanzia è espressa dall'art. 31 del codice penale dell'Albania.

La reclusione si considera una pena di carattere principalmente penale, ma durante l'esecuzione della stessa, si notano gli elementi educativi riguardanti il suo obiettivo, cioè il reinserimento sociale ${ }^{23}$. Penso che sia arrivato il momento di superare quest'idea e di non considerare il minore delinquente come un semplice soggetto del processo penale, il quale deve essere sottoposto alla condanna. II minore che infrange la legge e comette un reato è in fondo un essere umano ancora in via di sviluppo, un ragazzo o un adolescente che ha bisogno di cure speciali, che non potrà avere durante la reclusione.

La normativa internazionale a tutela dei minori mira all'obiettivo che l'imprigionamento deve essere una misura

\footnotetext{
${ }^{19}$ La creazione di queste sezioni è avvenuta con il decreto del Presidente della Repubblica n. 5351 del 11 giugno 2007. Attualmente, in Albania funzionano le sezioni penali minorili presso i Tribunali di Primo grado di 7 distretti giudiziali.

${ }^{20} \mathrm{Da}$ ora in poi citato come Regole dell'Avana.

${ }^{21}$ Articolo 16 della legge n. 8328 del 16 aprile 1998.

${ }^{22}$ Cfr. Shefqet Muçi, E drejta penale, pjesa e pergjithshme, Dudaj, Tiranë, 2007, pp. 238-241.

${ }^{23} \mathrm{Gli}$ stessi obiettivi sono stati definiti anche dalla Raccomandazione del CE (2003) 20, artt. 1, 2, 17 e 20.
} 
estrema e di una durata limitata (art. 37 della Convenzione sui Diritti dell'Infanzia, l'art. 19. 1 delle Regole di Pechino, artt. 2, 15 e 16 della Raccomandazione (87) 20 del CE, artt. 2, 3, 5, 9, 10 della Raccomandazione (2008) 11 del CE24). II codice penale albanese stabilisce che, per i minori di anni diciotto al momento in cui hanno commesso il reato, la pena di reclusione non può essere superiore alla metà della sanzione prevista dalla legge per il reato commesso (art. 51)25. L'art. 52 di questo codice stabilisce che il giudice, valutando la bassa pericolosità sociale del reato, le circostanze in cui è stato commesso e il comportamento precedente del minore, può astenersi dal pronunciare condanna. In questo caso, il giudice può disporre l'applicazione delle misure di sicurezza (collocamento in un riformatorio giudiziario). Con la legge $\mathrm{n}$. 10 023/2008 sono state apportate alcune modifiche al codice penale introducendo le sanzioni sostitutive alla pena detentiva. Queste sanzioni sono la semidetenzione, la sospenzione condizionale della pena, l'affidamento in prova e la detenzione domiciliare (artt. 58-59/a del codice penale dell'Albania). II legislatore non ha provvisto un apposito ordinamento nei confronti dei minori per non ricorrere alla carcerazione, in materia di sanzioni sostitutive alla pena detentiva.

L'art. 26 delle Regole di Pechino e gli artt. 79 e 80 delle Regole dell'Avana stabiliscono che le strutture nelle quali sono collocati i minori detenuti, dovrebbero avere una funzione educativa, communicativa e riabilitativa, affinchè siano in grado di avere un ruolo costruttivo e produttivo nella società ${ }^{26}$. La realtà albanese comunque si presenta diversa. Nella maggior parte dei casi, la punizione, l'esclusione, l'istituzionalizzazione, continuano a essere i modi per combattere la delinquenza minorile.

Per i minori di età inferiore ai quattordici anni, l'art. 46 del codice penale d'Albania prevede la sistemazione in un istituto di riabilitazione (riformatorio giudiziario), come misura di sicurezza. II giudice può disporre la sistemazione in tale istituto anche nel caso del perdono giudiziale ${ }^{27}$. Tale misura di sicurezza è stata prevista dalla legge, pero in prattica è inapplicabile. Durante il regime totalitario, nel dicembre del 1986 in Albania ha cominciato a funzionare la scuola di riabilitazione dei minori28. In questa scuola erano collocati minori delinquenti della fascia d'età dai $14-18$ anni, di sesso maschile, dichiarati colpevoli dal tribunale e altri non imputabili, minori di quattordici anni che avevano commesso dei reati. I minori collocati in questa scuola erano sottoposti a un programma d'istruzione obbligatoria e a lavori diversi, soprattutto nel settore agricolo. La scuola ha cessato di funzionare nel 1991 dopo la caduta del sistema. Attualmente, in Albania non esistono le strutture riconosciute dallo Stato nelle quali si possano eseguire le misure di sicurezza e il tribunale per questa ragione non le provvede.

Riguardo al minore detenuto, l'art. 37/c della Convenzione sui Diritti dell'Infanzia stabilisce che qualsiasi fanciullo privato della libertà deve essere detenuto separato dagli adulti, a meno che la soluzione contraria non sia considerata preferibile nell'interesse superiore del fanciullo. Lo stesso principio è espresso dall'art. 26.3 delle Regole di Pechino e dall'art. 29 delle Regole dell'Avana. Questi atti richiedono l'istituzione di strutture speciali penitenziarie, ove ci siano solo minori (art. 40, comma 3, della Convenzione sui Diritti dell'Infanzia). Tale principio è stato rispettato dalla legislazione penale albanese, per l'appunto dall'art. 33 del codice penale, dall'art. 5 della legge n. 8331/98 "Esecuzione delle pene", dall'art. 17 della legge n. 8328/98 "I diritti e il trattamento dei detenuti". Comunque, un vero istituto penitenziario per i minorenni, situato a Kavaja, funziona in Albania solo dall'ottobre del 2009 e serve come centro di riabilitazione, consulenza e formazione per i minori autori di reato dell'età dai quattordici fino ai diciotto anni che scontano la pena di reclusione. L' $80 \%$ del personale di questo istituto è specializzato nelle scienze umane, mentre la parte rimanente appartiene alla polizia dello stato. Gli ambienti sono adattati in modo tale che l'isolamento si percepisca in meno possibile e si svolgono attività educative e di natura sociale e psicologica, con l'obiettivo di riabilitare i minori. Negli anni 2008-2009 hanno iniziato a funzionare cinque istituti penitenziari, con apposite sezioni per i minori detenuti29.

In conformità agli artt. 27 e 28 delle Regole dell'Avana, l'art. 10 della legge albanese n. 8328/1998 stabilisce che i detenuti minorenni devono essere trattati in maniera personalizzata a secondo delle circostanze psicologiche e sociali.

Nello stesso modo, l'art. 37 della stessa legge, in conformità all'art. 38 delle Linee guida di Riyadh, stabilisce il diritto d'istruzione dei minori detenuti, a favore della loro riabilitazione. Una scuola elementare (di nove anni di durata)

\footnotetext{
${ }^{24} \mathrm{La}$ disposizione penale stabilisce la riduzione della pena di reclusione e non di quella pecuniaria. Quest'ultima si infligge in modo uguale sia per gli adulti che per i minori.

${ }^{25}$ Lo stesso principio è stabilito dagli artt. 50, 53, 78 della Racc. (2008) 11 "Norme europee per i minori autori di reato destinatari di sanzioni o misure".

${ }^{26} \mathrm{v}$ sopraccitato.

${ }^{27}$ La scuola è stata istituita con decreto $\mathrm{n}$. 7003 del 28.10.1985 del Presidium dell'Assemblea Nazionale e la delibera del Consiglio dei Ministri n. 438 del 20.12.1986.

${ }^{28}$ v www.dpbsh.gov.al voce Sistemi Penitenciar.

${ }^{29}$ v. www.dpbsh.gov.al, voce Sistemi Penitenciar.
} 
funziona dal 2000 presso l'istituto penitenziario di Vaqarr, Tirana ${ }^{30}$.

I minori detenuti devono avere contatti continui con il mondo esterno, con i genitori e famigliari. (art. 37/c della Convenzione sui Diritti dell'Infanzia, art. 26.5 delle Regole di Pechino, art. 59 delle Regole dell'Avana). Tale diritto è riconosciuto al detenuto minorenne anche dalla legge albanese n. 8328/1998, artt. 32, comma 4, e 40.

L'art. 41 delle Regole dell'Avana raccomanda di facilitare ai minori l'accesso a una biblioteca con libri e altre pubblicazioni che siano educativi e ricreativi ${ }^{31}$, di informarsi tramite i giornali, le riviste, la televisione, ecc., (art. 62); di esercitare attività religiose e spirituali (art. 48). Questi diritti sono riconosciuti ai minori detenuti anche dalla legislazione albanese, concretamente dagli artt. 37, comma 3, e 32, comma 2, della legge n. 8328/1998.

La $5^{\circ}$ sezione delle Regole dell'Avana presta attenzione alla formazione del personale che prenderà cura di questi minori, il quale dovrebbe essere in grado di affrontare i loro problemi e di includere figure tali come educatori, istruttori professionali, assistenti, assistenti sociali, psichiatri e psicologi. L'art. 32, comma 3, della legge albanese n. 8328/1998 sancisce che il personale dell'amministrazione penitenziaria deve essere addestrato nel campo dell'insegnamento.

\section{Conclusioni}

Lo stato albanese ha cercato dopo gli anni '90 di adottare una legislazione a tutela dei diritti umani mostrando particolare attenzione anche ai diritti dei minori. Rivolgendosi alla legislazione penale si notano i primi passi verso la nascita del sistema penale minorile. A questo scopo sarebbe necessario di:

- Costruire un sistema differenziato di diritto penale minorile che tende alla tutela dei minori in conformità alla normativa internazionale come la Convenzione ONU sui Diritti dell'Infanzia, le Regole di Pechino, le Linee Guida di Riyadh, le Regole dell'Avana e della normativa europea a tutela dei minori;

- Applicare delle misure alternative alla detenzione cercando di minimizzare l'imprigionamento del minore come il miglior modo per tutelare il suo superiore interesse;

- Specializzare tutti i soggetti istituzionali che operano nel processo minorile, giudici, publici ministeri, ufficiali della Polizia Giudiziaria, avvocati, ecc.;

- Creare istituzioni di riabilitazione speciale per i minori di anni quattordici che hanno commesso dei reati;

- Provvedere delle misure a favore del reinserimento sociale dei minori condannati, dopo il periodo della reclusione.

\section{Bibliografia}

\section{Fonti archivistiche:}

\section{Legge Penale Ottomana}

Codice Penale del Regno d'Albania del 1928

L. 24 dicembre 1946, n. 253, in materia di "Disposizioni Penali Generali"

Codice Penale della Repubblica d'Albania del 1948

Codice Penale della Repubblica Popolare d'Albania del 1952

Codice Penale della Repubblica Popolare Socialista d'Albania del 1977

\section{Atti normativi della Repubblica d'Albania:}

Costituzione della Repubblica d'Albania del 1998, aggiornata

Codice Penale della Repubblica d'Albania del 1995, aggiornato

Codice di Procedura Penale della Repubblica d'Albania del 1995, aggiornato

L. 21 aprile 1998, n. 8331, in materia di "Esecuzione delle sentenze penali", aggiornato

L. 16 aprile 1998, n. 8328, in materia di "Diritti e trattamento dei detenuti", aggiornato

L. 26 giugno 2003, n. 9090, in materia di "La mediazione finalizzata alla conciliazione delle controversie"

\footnotetext{
${ }^{30}$ Nell'istituto penitenziario per i minorenni di Kavaja questa regola si sta applicando dal personale. In precedenza, ai minori detenuti non era offerto un servizio del genere.
}

${ }^{31}$ This figure is based on random assumption with yearly average growth rate of $2 \%$. 


\section{Normativa internazionale:}

Convenzione ONU sui Diritti dell'Infanzia del 1989

Regole Minime delle Nazioni Unite sull'Amministrazione della Giustizia Minorile (c.d. Regole di Pechino) 29.11.1985

Linee Guida delle Nazioni Unite sulla Prevenzione della Delinquenza Minorile ( c.d. Linee Guida di Riyadh) 14.12.1990

Regole delle Nazione Unite per la Protezione dei Minori Privati della Libertà (c.d. Regole dell'Avana) 14.12.1990

Convenzione Europea per la Salvaguardia dei Diritti dell'Uomo e delle Libertà Fondamentali 04.11.1950

Raccomandazione (87) 20 in materia di "Reazioni sociali alla delinquenza minorile"

Raccomandazione (2006) 2 - Regole penitenziarie europee - 11 gennaio 2006

Raccomandazione (2008) 11 - Norme europee per i minori autori di reato destinatari di sanzioni o misure

\section{Riferimenti bibliografici:}

Corte Costituzionale, 28 febbraio 2000, n. 7, in Vendime të Gjykatës Kushtetuese të RSH, Tiranë, Mihal Duri e Re, 2002. ISBN 99927708-0-5

Shefqet Muçi, E drejta Penale, Pjesa e përgjithshme, Tiranë, Dudaj, 2007. ISBN 978-99943-0-029-7

\section{Pagine web consultate:}

www.ligjet.org

www.qbz.gov.al

www.gjykataelarte.gov.al

www.dpbsh.gov.al

https://wcd.coe.int 